BMJ Paediatrics Open

\title{
Family talk intervention in paediatric oncology: a pilot study protocol
}

Malin Lövgren,, ${ }^{1,2}$ Ulrika Kreicbergs, ${ }^{1,2}$ Camilla Udo ${ }^{1,3}$

To cite: Lövgren $\mathrm{M}$, Kreicbergs U, Udo C. Family talk intervention in paediatric oncology: a pilot study protocol. BMJ Paediatrics Open 2019;3:e000417. doi:10.1136/ bm.jpo-2018-000417

Received 14 December 2018 Revised 20 December 2018 Accepted 21 December 2018

Check for updates

(c) Author(s) (or their employer(s)) 2019. Re-use permitted under CC BY-NC. No commercial re-use. See rights and permissions. Published by BMJ.

${ }^{1}$ Department of Health Care Sciences, Palliative Research Centre, Ersta Sköndal Bräcke University College, Stockholm, Sweden

${ }^{2}$ The Department of Women's and Children's Health, Childhood Cancer Research Unit,

Karolinska Institutet, Stockholm Sweden

${ }^{3}$ School of Education, Health and Society, Dalarna University, Falun, Sweden

Correspondence to Malin Lövgren; malin.lovgren@ esh.se

\section{ABSTRACT}

Introduction There is evidence that families with a child diagnosed with cancer need psychosocial support throughout the illness trajectory. Unfortunately, there is little research into psychosocial interventions for such families, especially interventions where the entire family is involved. The aim of this pilot study is therefore to evaluate a psychosocial intervention, the family talk intervention (FTI), in paediatric oncology in terms of study feasibility and potential effects.

Methods and analysis This pretest/post-test intervention pilot study is based on families with a child diagnosed with cancer. All families that include at least one child aged $6-19$ years (ill child and/or sibling) at one of the six paediatric oncology centres in Sweden between September 2018 and September 2019 will be asked about participation. The intervention consists of six meetings with the family (part of the family or the entire family), led by two interventionists. The core elements in the intervention are to support the families in talking about the illness and related subjects, support the parents in understanding the needs of their children and how to support them and support the families in identifying their strengths and how to use them best. Mixed methods are used to evaluate the intervention (web-based questionnaires, interviews, field notes and observations). Self-reported data from all family members are collected at baseline, directly after the intervention and 6 months later. Study outcomes are family communication, knowledge about the illness, resilience, quality of life and grief.

Ethics and dissemination The study has been approved by the Regional Ethical Review Board in Stockholm (Dnr 2018/250-31/2 and 2018/1852-32). Data are processed in coded form, accessible only to the research team and stored at Ersta Sköndal Bräcke University College in a secure server.

Trial registration ClinicalTrials.gov Identifier NCT03650530, registered in August 2018.

\section{INTRODUCTION}

Childhood cancer is a life-threatening illness that involves life changes not only for the ill child but also for the rest of the family. Studies show that children with cancer experience various forms of distress associated with the cancer experience, for example, feeling lonely, isolated and powerless. ${ }^{12}$ Much data indicate that child functioning is closely associated with and often dependent on parent and family functioning. ${ }^{3}$ Parents play a significant

\section{What is already known on this topic?}

- There is evidence and a strong recommendation that paediatric oncology centres should provide psychosocial support for families throughout the illness trajectory.

- Family health can be maintained and supported if interventions encompass both each individual family member and the family as a unit.

- There is little research about psychosocial interventions that includes entire families in paediatric oncology, and none have been evaluated in Sweden yet.

\section{What this study hopes to add?}

An evaluation of a psychosocial intervention in paediatric oncology in terms of study feasibility and potential effects.

- Improved family communication, knowledge about the illness, quality of life, grief and resilience among family members.

- Making all family members' experiences heard (parents, ill and healthy children) in the evaluation of a psychosocial intervention for families in paediatric oncology.

role in providing support throughout a child's development, to both healthy and ill children, but parents of children with cancer are at risk for marked or prolonged distress or psychopathology. ${ }^{4}$ Siblings have reported difficulties dealing with their parents' and the ill child's suffering, loneliness in relation to their own feelings, as well as uncertainty regarding their brother's/sister's treatment and prognosis. ${ }^{5}$ Research has concluded that it is also important for healthy siblings to be seen and to be given attention. ${ }^{6}$ Empirical studies have identified family-related and care-related factors as important for the psychological well-being of all family members during illness and after death. For example, medical information and family communication influenced long-term psychological morbidity. ${ }^{78}$

There is evidence and a strong recommendation that paediatric oncology centres should provide psychosocial support for 
families throughout the illness trajectory. ${ }^{4910}$ Nevertheless, such services vary widely across centres. ${ }^{910}$ In the case of illness in the family, family health can be maintained and supported if interventions encompass both each individual family member and the family as a unit, that is, if the intervention is based on the family as a system. ${ }^{11}$ A systematic review of psychosocial interventions for childhood cancer identified 21 studies between 1980 and 2008, of which only six involved entire families. ${ }^{12}$ The majority of interventions reviewed were associated with positive effects for participants, even if methodological considerations were highlighted by the authors, for example, sample size, that only one member of the family was evaluated or that mixed methods were poorly used in the evaluation. ${ }^{12}$ Steel $e t a l,{ }^{9}$ in a systematic review that encompassed later studies (between 1995 and 2015), found 19 papers that described a psychosocial intervention in paediatric oncology. Only one of those involved entire families. ${ }^{13}$ In a Swedish systematic review, based on nursing and psychosocial research in paediatric oncology during the period 2000-2013, it was found that only six out of 137 studies included an intervention, and none involved all family members. ${ }^{14}$ Researchers highlight that there is a need for further research and development of psychosocial interventions for families in paediatric oncology. ${ }^{9} 1214$

In recent years, a family-based intervention for families with children in which one parent has depression, ${ }^{15}$ called the Beardslee's Family Intervention or the family talk intervention (FTI), has begun to be used clinically in Sweden. Research concerning this intervention has increased in both psychiatry ${ }^{17}$ and somatic care, ${ }^{18-21}$ although further evaluations are needed. Unfortunately, no psychosocial family-based intervention in paediatric oncology for all family members has yet been evaluated in Sweden, despite the urgent need for psychosocial support for entire families. The aims of this pilot study are therefore to evaluate whether the FTI is feasible in paediatric oncology and to explore potential effects of the FTI.

\section{METHODS AND ANALYSIS}

\section{Study design and participants}

This is a pretest/post-test pilot intervention study with a mixed methods design. The intervention consists of a family-based support programme for families with a child with cancer. The families are recruited from one of the six paediatric oncology centres in Sweden between September 2018 and September 2019. Eligible families are those with at least one child (ill or healthy sibling) between 6 and 19 years old and whose ill child is treated for cancer at this oncology centre during this time period. One inclusion criteria is also that $2-3$ months should have passed since diagnosis or relapse. The entire family or a part of the family can participate in the intervention, but a minimum is that at least one parent/guardian and one child aged 6-19years participate. The participating family members have to understand and speak Swedish. Nurses and counsellors at the clinic identify families that meet the inclusion criteria. The families are thereafter contacted by phone by one of the two interventionists (one social worker and one deacon), who are educated in conducting the FTI, to set up meetings where the families are given verbal and written information about the study. Those who are willing to participate are asked for informed consent.

\section{The FTI}

The FTI was originally developed for families with a parent with an affective disorder and children aged $6-18$ years. ${ }^{1522} 23$ It is not a psychotherapeutic intervention. The overarching aims of the intervention are to support an open, honest family communication and to increase family members' understanding of the disease. The core elements are to support the families in talking about the illness and related subjects, support the parents in understanding the needs of their children and how to support them, and support the families in identifying their strengths and how to use them best. By promoting protective factors, a process that builds up resilience is initiated. The FTI has its main focus on the children and has an eclectic approach that includes psychoeducative, narrative and dialogical ways of working. The psychoeducative element focuses on increased knowledge about the illness and related subjects and the narrative element involves the family's own stories. The dialogical way of working focuses on making problematic situations visible by making the children's voices heard, sharing experiences within the family and seeing all the family members' different perspectives. 152223

The FTI is led by two interventionists specially educated in the FTI, who work as a pair and both have long clinical experience of working with families affected by life-threatening illnesses. The FTI is manual based and the interventionists will follow a structured protocol, in which they will also take notes.

The FTI entails six meetings at intervals of 1-2 weeks (table 1). The meetings with each family are held in a place chosen by that family. If the intervention is unexpectedly interrupted and cannot be finished as scheduled due to special circumstances, extra meetings will be available (meetings 7-11).

\section{Meetings 1-2}

Include only the parent(s)/guardian(s) and focus on their experiences of the situation and the consequences of the cancer diagnosis for each family member. During the meeting, each child's situation will be discussed, including strengths, problems, worries, the situation in school and with friends, their social network and the child's knowledge of the disease. The parent(s)/guardian(s) will formulate the goal of the intervention. 
Table 1 Involved family members and focus of each meeting in the family talk intervention.

\begin{tabular}{|c|c|c|}
\hline Meeting & Involved family members & Focus of the meeting \\
\hline $1-2$ & The parents/guardians & $\begin{array}{l}\text { The parents' history. Setting up goals for the } \\
\text { intervention. }\end{array}$ \\
\hline $\begin{array}{l}3 \text { (several meetings if there is more } \\
\text { than one child in the family) }\end{array}$ & $\begin{array}{l}\text { Each child (preferably without } \\
\text { parents/guardians) }\end{array}$ & $\begin{array}{l}\text { The child's understanding of the illness and the } \\
\text { situation. The child's worries and questions. }\end{array}$ \\
\hline 4 & The parents/guardians & $\begin{array}{l}\text { Summary of worries and questions from meeting } 3 . \\
\text { Planning 'the family talk' (meeting 5). }\end{array}$ \\
\hline 5 & The entire family & $\begin{array}{l}\text { The family talk. Preferably led by the parents and } \\
\text { consisting of questions from both children and parents. }\end{array}$ \\
\hline 6 & The entire family & $\begin{array}{l}\text { Follow-up with a focus on how to communicate within } \\
\text { the family in the future to achieve the family's goals. }\end{array}$ \\
\hline $7-11$ & & Extra meetings. \\
\hline
\end{tabular}

\section{Meeting 3}

Interviews will be held with each child, preferably without the parent(s)/guardian(s), concerning the child's life situation, feelings, understanding of the disease, questions and hobbies. The relationship with their parent(s)/ guardian(s) is discussed, as is the child's social network. The interventionist(s) identifies protective factors from the child's narrative (eg, well-functioning school life and relationships with friends), as well as risk factors (eg, poor social network). The child can also formulate questions for the parent(s)/guardian(s) and what he/she wants to discuss during the family meeting.

\section{Meeting 4}

Includes the parent(s)/guardian(s) and focuses on planning the family meeting. The children's thoughts and questions serve as a guide for the upcoming family meeting.

\section{Meeting 5}

Is preferably led by the parent(s)/guardian(s), to facilitate continuous communication within the family and consists of questions and issues raised earlier by the family members. This family meeting can be seen as a starting point for communication within the family.

\section{Meeting 6}

Is a follow-up with all family members, preferably held within a month of meeting 5 . The meeting is guided by the family members' needs, for example, regarding communication and parenting.

\section{Measurements}

Mixed methods, ${ }^{24}$ including web-based questionnaires (table 2), face-to-face interviews, field notes taken by the interventionists and observations, are used to evaluate the study feasibility and potential effects of the FTI. Data are collected at baseline, directly after the intervention has ended (follow-up 1) and 6 months later (follow-up 2).

\section{Main outcome}

Self-reported family communication among all family members.

\section{Secondary outcome}

Self-reported knowledge about the illness, resilience, quality of life and grief experiences associated with the illness.

The outcomes are chosen based on the goals of the intervention and in accordance with the literature on psychosocial support programmes in paediatric oncology, ${ }^{9} 1214$ family responses to family interventions in healthcare ${ }^{25}$ and an earlier FTI study in palliative care. ${ }^{21}$

\section{Questionnaire data}

In addition to family background characteristics, the following areas are measured in the web-based questionnaires.

\section{About the FTI: expectations and experiences of participation}

At baseline, the family members are asked about their expectations of and concerns regarding the FTI, as well as the reasons for participation (parents/guardians and children aged 13 and older). At follow-up 1, questions about the following areas are included: the length and number of meetings, the timing of the FTI, if their expectations have been met or not, if the FTI has help the family, if they have felt understood by the interventionists and if they have felt that they could talk openly during the meetings. They will also be asked about potential improvements to the FTI, as well as whether or not they would recommend the FTI. At follow-up 2, the family members are asked to describe if and how the FTI has helped them during the last 6 months.

\section{Family communication}

The standardised instrument Family Communication from Family Adaptability and Cohesion Scale IV (FACES IV) is used for parents/guardians and for children aged 13 and older. ${ }^{26}{ }^{27}$ The instrument focuses on exchange of information, both factual and emotional. It covers the constraints and degree of understanding and satisfaction experienced in family communication interactions. In addition, study-specific questions from an FTI study in another context ${ }^{21}$ and earlier nationwide surveys ${ }^{28} 29$ are used, for all family members. 


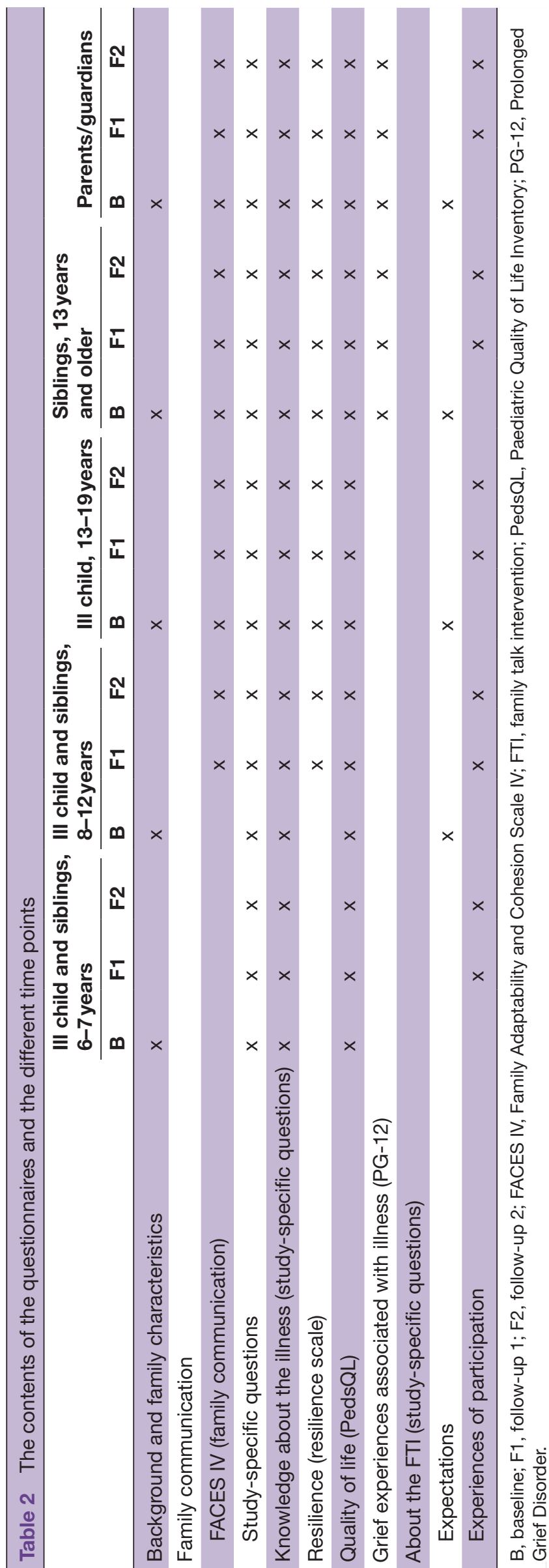

\section{Knowledge about the illness}

This is assessed with study-specific questions from an FTI study in another context ${ }^{21}$ and earlier nationwide surveys ${ }^{28}{ }^{29}$ for all family members.

\section{Resilience}

The short version of the Resilience Scale, RS-14, is used for parents/guardians and for children 13years and older. ${ }^{30}{ }^{31}$ It includes the following domains: self-reliance (the belief in oneself and one's capabilities), purpose (meaningfulness in life), equanimity (a balanced perspective of one's life), perseverance (the ability to keep going despite setbacks) and authenticity (the recognition of one's unique path and acceptance of one's life). The Resilience Scale for Children (RS-10) is used for children $8-12$ years and includes the same domains as for adults. ${ }^{32}$

\section{Quality of life}

Quality of life for ill children and siblings, in all age groups, is assessed using the standardised instrument Paediatric Quality of Life Inventory V.4.0, which includes the following domains: physical functioning, emotional functioning, social functioning and school functioning. ${ }^{33-35}$

\section{Grief experiences associated with illness}

This is measured with Prolonged Grief Disorder (PG-12) among parents/guardians and siblings 13years and older. ${ }^{36}$ It covers: (1) separation distress, which is characterised by manifestations of longing and yearning, (2) emotional, cognitive and behavioural symptoms, for example, diminished sense of self, feeling stunned or shocked by the patient's illness, having trouble accepting it, and feelings of meaninglessness and (3) social and occupational functioning.

\section{Pretest of the study-specific questionnaires}

Seven study-specific web-based questionnaires have been developed: one parent/guardian version, three ill child versions (6-7years, 8-12 years, 13-19years) and three sibling versions (6-7years, 8-12years and 13 years and older). To pretest the questionnaires, five families (three bereaved and two non-bereaved) were asked to answer the web-based versions and give feedback on the questions. Based on the feedback, the structure of the adult questionnaire was changed, the versions for the youngest children were shortened and some minor stylistic changes were made.

\section{Interview and observational data}

Interviews with the families are conducted after meeting 6 . The focus of the interviews is the families' experiences of participating in the FTI and the study outcomes, for example, family communication. Observations, such as field notes taken by one of the interventionists at every meeting with the families, in accordance the FTI manual, will cover the content in each meeting, for example, the families' goals with the FTI, which family members participated in each meeting, conversation topics, special 
events during the meetings, etc. Such data allow us to examine, for example, if the children's expressed needs/ issues can be related to the family's goal with the FTI or if the FTI helped the family with these problems. In addition, notes will be taken during the study process on recruitment of families, including families' willingness to participate, the amount of missing data and dropouts.

\section{Family and public involvement}

The non-profit organisation, the Swedish Childhood Foundation, financed the project after reviewing the project plan. Two parents with experiences from childhood cancer were involved in designing the study. Later, five families tested and gave feedback on the questionnaires (see above). The Swedish organisation for the Beardslee's Family Intervention has been involved throughout the study for guidance regarding the FTI. The burden of the intervention and time required to participate in the research is one of the outcomes tested in this pilot study.

\section{Power calculation}

Knowledge about how many families want to participate during the course of a year at one paediatric oncology centre will provide an estimate of how many families could be willing to participate each year in a future main study. In addition, we will during a 1-year period have the opportunity to evaluate the data collection methods, for example, the distribution of the web-based questionnaires, and the amount of missing data and dropouts, which are highly important for planning a future main trial. A future main trial, with a cluster randomised design, should preferably be based on some or all six paediatric oncology centres in Sweden. A power calculation for a future trial with regard to the main outcome (with $80 \%$ power, $5 \%$ significance level, two-sided t-test and a SD based on previous studies using the same instrument) estimates a sample size of 128 families (64 families in each group), based on the minimum required difference in terms of family communication using FACES IV.

\section{Data analysis}

Interviews and field notes taken by the interventionists will be analysed with content analysis ${ }^{38}$ and the study process will be observed. As this is the first FTI in paediatric oncology, the results regarding potential effects of the intervention will indicate the direction of further studies; data from the questionnaires will be explored with descriptive statistics focusing on changes over time, for example, regarding family communication.

\section{Ethics and dissemination}

The study has received ethical approval from the Regional Ethical Review Board in Stockholm (Dnr 2018/250-31/2 and 2018/1852-32). Data are processed in coded form and accessible only to the research team. All person-linked data are stored at Ersta Sköndal Bräcke University College and in a secure server. Only coded data will be used in the analyses. Data will be presented in such a way that no specific family/person can be identified. We hope this study will be worth testing nationwide and that the FTI, in the long run, will contribute to increased family communication and well-being for families in paediatric oncology.

Acknowledgements We want to thank the Swedish organisation for the Beardslee's Family Intervention for sharing their experiences with us.

Contributors ML has contributed to the design of the study, pretest of the questionnaires and writing the manuscript. UK and CU have contributed to the design of the study and edited the manuscript. All authors have read and approved the final version.

Funding This work was supported by the Swedish Childhood Foundation, grants number TJ2016-005 and PR2016-0013.

Competing interests None declared.

Patient consent for publication Not required.

Provenance and peer review Not commissioned; externally peer reviewed.

Open access This is an open access article distributed in accordance with the Creative Commons Attribution Non Commercial (CC BY-NC 4.0) license, which permits others to distribute, remix, adapt, build upon this work non-commercially, and license their derivative works on different terms, provided the original work is properly cited, appropriate credit is given, any changes made indicated, and the use is non-commercial. See: http://creativecommons.org/licenses/by-nc/4.0/.

\section{REFERENCES}

1. Darcy L, Björk M, Enskär $\mathrm{K}$, et al. The process of striving for an ordinary, everyday life, in young children living with cancer at six months and one year post diagnosis. Eur J Oncol Nurs 2014;18:605-12.

2. Darcy L, Knutsson S, Huus K, et al. The everyday life of the young child shortly after receiving a cancer diagnosis, from both children's and parent's perspectives. Cancer Nurs 2014;37:445-56.

3. Mavrides N, Pao M. Updates in paediatric psycho-oncology. Int Rev Psychiatry 2014;26:63-73.

4. Kearney JA, Salley CG, Muriel AC. Standards of psychosocial care for parents of children with cancer. Pediatr Blood Cancer 2015;62:S632-S683.

5. Long KA, Marsland AL, Wright A, et al. Creating a tenuous balance: siblings' experience of a brother's or sister's childhood cancer diagnosis. J Pediatr Oncol Nurs 2015;32:21-31.

6. Long KA, Lehmann V, Gerhardt CA, et al. Psychosocial functioning and risk factors among siblings of children with cancer: An updated systematic review. Psychooncology 2018;27:1467-79.

7. Kreicbergs $U$, Valdimarsdóttir U, Onelöv E, et al. Anxiety and depression in parents 4-9 years after the loss of a child owing to a malignancy: a population-based follow-up. Psychol Med 2004;34:1431-41.

8. Wallin AE, Steineck G, Nyberg T, et al. Insufficient communication and anxiety in cancer-bereaved siblings: A nationwide long-term follow-up. Palliat Support Care 2016;14:488-94.

9. Steele AC, Mullins LL, Mullins AJ, et al. Psychosocial interventions and therapeutic support as a standard of care in pediatric oncology. Pediatr Blood Cancer 2015;62:S585-S618.

10. Gerhardt CA, Lehmann V, Long KA, et al. Supporting siblings as a standard of care in pediatric oncology. Pediatr Blood Cancer 2015;62:S750-S804.

11. Wright LM, Leahey M. Nurses and families: a guide to family assessment and intervention. 6 edn. Philadelphia: FA: Davis Company, 2013.

12. Meyler E, Guerin S, Kiernan G, et al. Review of family-based psychosocial interventions for childhood cancer. J Pediatr Psychol 2010;35:1116-32.

13. Kazak AE, Alderfer M, Rourke MT, et al. Posttraumatic stress disorder (PTSD) and posttraumatic stress symptoms (PTSS) in families of adolescent childhood cancer survivors. J Pediatr Psychol 2004;29:211-9.

14. Enskär K, Björk M, Knutsson S, et al. A Swedish perspective on nursing and psychosocial research in paediatric oncology: $A$ literature review. Eur J Oncol Nurs 2015;19:310-7.

15. Beardslee WR, Gladstone TR, Wright EJ, et al. A family-based approach to the prevention of depressive symptoms in children at risk: evidence of parental and child change. Pediatrics 2003;112:e11 9-e131. 
16. Pihkala H, Sandlund M, Cederström A. Children in Beardslee's family intervention: relieved by understanding of parental mental illness. Int $J$ Soc Psychiatry 2012;58:623-8.

17. Pihkala $H$, Sandlund $M$, Cederström A. Initiating communication about parental mental illness in families: an issue of confidence and security. Int J Soc Psychiatry 2012;58:258-65.

18. Niemelä M, Repo J, Wahlberg KE, et al. Pilot evaluation of the impact of structured child-centered interventions on psychiatric symptom profile of parents with serious somatic illness: struggle for life trial. J Psychosoc Oncol 2012;30:316-30.

19. Bugge KE, Helseth S, Darbyshire P. Parents' experiences of a family support program when a parent has incurable cancer. J Clin Nurs 2009;18:3480-8.

20. Bugge KE, Helseth S, Darbyshire P. Children's experiences of participation in a family support program when their parent has incurable cancer. Cancer Nurs 2008;31:426-34.

21. Eklund R, Kreicbergs U, Alvariza A, et al. The family talk intervention in palliative care: a study protocol. BMC Palliat Care 2018;17:35.

22. Beardslee WR, Swatling S, Hoke L, et al. From cognitive information to shared meaning: healing principles in prevention intervention. Psychiatry 1998;61:112-29.

23. Focht L, Beardslee WR. "Speech after long silence": the use of narrative therapy in a preventive intervention for children of parents with affective disorder. Fam Process 1996;35:407-22.

24. Creswell JW. A concise introduction to mixed methods research. USA: Sage Publication Inc, 2015:132.

25. Ostlund U, Persson $\mathrm{C}$. Examining family responses to family systems nursing interventions: an integrative review. J Fam Nurs 2014;20:259-86.

26. Olson D. Faces iv and the circumplex model: validation study. $J$ Marital Fam Ther 2011;37:64-80.
27. Marsac ML, Alderfer MA. Psychometric properties of the FACES-IV in a pediatric oncology population. J Pediatr Psychol 2011;36:528-38.

28. Kreicbergs $U$, Valdimarsdóttir $U$, Onelöv E, et al. Talking about death with children who have severe malignant disease. N Engl J Med 2004;351:1175-86.

29. Lövgren $M$, Jalmsell L, Eilegård Wallin A, et al. Siblings' experiences of their brother's or sister's cancer death: a nationwide follow-up 2-9 years later. Psychooncology 2016;25:435-40.

30. Lundman B, Strandberg G, Eisemann M, et al. Psychometric properties of the swedish version of the resilience scale. Scand $J$ Caring Sci 2007;21:229-37.

31. Wagnild GM, Young HM. Development and psychometric evaluation of the resilience scale. J Nurs Meas 1993;1:165-78.

32. Wagnild G. Resilience Scale for Children (RS10) user's guide. USA: The Resilience Center, 2015.

33. Varni JW, Burwinkle TM, Seid M, et al. The PedsQL 4.0 as a pediatric population health measure: feasibility, reliability, and validity. Ambul Pediatr 2003;3:329-41.

34. Varni JW, Seid M, Kurtin PS. PedsQL 4.0: reliability and validity of the Pediatric Quality of Life Inventory version 4.0 generic core scales in healthy and patient populations. Med Care 2001;39:800-12.

35. Varni JW, Seid M, Rode CA. The PedsQL: measurement model for the pediatric quality of life inventory. Med Care 1999;37:126-39.

36. Prigerson HG, Horowitz MJ, Jacobs SC, et al. Prolonged grief disorder: psychometric validation of criteria proposed for DSM-V and ICD-11. PLoS Med 2009;6:e1000121.

37. Pohlkamp L, Kreicbergs U, Prigerson HG, et al. Psychometric properties of the prolonged grief disorder-13 (pg-13) in bereaved swedish parents. Psychiatry Res 2018;267:560-5.

38. Hsieh HF, Shannon SE. Three approaches to qualitative content analysis. Qual Health Res 2005;15:1277-88. 